\title{
A Novel Locust-inspired Jumping Robot Driven by Pneumatic Muscle Actuators
}

\author{
Jun Zhong ${ }^{1, \text { a }}$,Chun Zhao ${ }^{1, b}$ and Hui Su ${ }^{1, c}$ \\ ${ }^{1}$ College of Mechanical and Electrical Engineering, Hohai University, Changzhou 213022, Jiangsu \\ Province, P.R. China \\ azhongjun@hhu.edu.cn, b171318010038@hhu.edu.cn, c1146769120@qq.com
}

Keywords: jumping robot, locusts, bionics, pneumatic muscle actuators, simulation.

Abstract. Jumping robots have been a hot research spot, since they are playing an important role in the modern wars, aviation and exploration, due to their good ability in obstacle surmounting, excellent adaptability to the ground and strong adaptability to the environment. This paper presents a novel bionic locust jumping robot driven by pneumatic muscle actuators. Dynamic simulation in Adams software is carried out to validate the scheme of the robot. The simulation results prove the feasibility of the design, and provide a reference for the selection of pneumatic muscles.

\section{Introduction}

Jumping motions have obvious advantages in uneven physiognomy or rugged environment compared to crawl and walking mode. Therefore, many jumping robots have been being developed in recent years [1-16]. M Kovač et.al proposed a novel spherical robot with the function of jump, recovery and orienting itself [1]. K Hosoda et.al studied human muscular skeleton mechanism and designed a humanoid jumping robot driven by pneumatic muscle actuators[2]. T Wang proposed a CPG control mechanism for hopping motion of biped robot in unpredictable circumstances [3]. In the research, the motion control task is divided into motion sequence control and output force control. J Zhao presented a miniature jumping robot named Michigan State University Jumper, which showed the capacity in continuous steerable jumping and self-righting and steering [4]. M Reis explored a robot with the function of hopping by free vibration of an elastic curved beam. His robot consisted of a specifically shaped elastic curved beam and a small rotating mass [5]. JS Koh devised a flea-inspired catapult mechanism which was categorized as an active storage and active release elastic catapult [6]. FB Mathis adopted the spring loaded inverted pendulum model to investigate the control strategy of a hopping robot [7]. In this paper, a locust-inspired jumping robot driven by pneumatic muscles are developed. Mechanical structure is firstly introduced and then simulation is performed to validate the jumping capacity of the robot.

\section{The mechanism design of a locust-inspired jumping robot}

Analysis of the jumping mechanism of locusts. As the main component of jumping motion, the locusts' hind legs can achieve the length of more than $40 \mathrm{~mm}$ if completely extends, and three times more than lengths of middle legs and fore legs. Hind leg consists of two muscles: one is the tibial extensor muscle, and the other is the flexor muscle. The former realizes leg extension, and the latter is responsible of leg contraction. The femora are connected with the tibia, which can rotate in a certain direction, so that the tibia and the femora are contracted or stretched together. Oblique muscles in the femora operate movements of tibia.

Mechanism design of the robot. By analyzing the structure and degree of freedoms, locusts' hind legs can be abstracted to be a planar bar mechanism with three DOFs, i.e. hip joint, knee joint, and ankle joint owns single DOF, respectively. In Figure 1, $\theta_{1}$ represents the angle between the femora and vertical direction, $\theta_{2}$ is the angle between the femora and the tibia, and $\theta_{3}$ the angle between the tibial and the toe end. 
According to the scheme in Figure 1, the locust-inspired jumping robot is devised and shown in Figure 1. The hind legs of the bionic locust jumping robot are of parallelogram configuration. This type of four bar mechanism can imitate the jumping action of the biological locust when the rod 4 is driven.

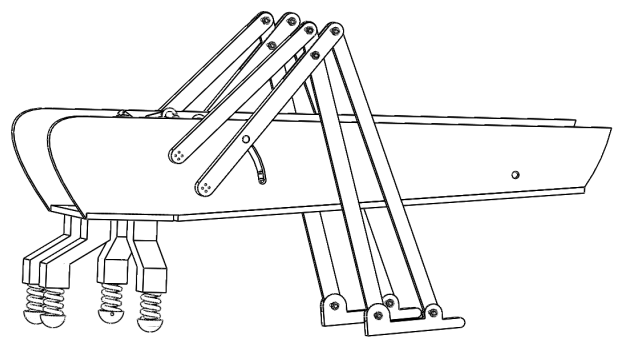

Figure 1 The locust-inspired jumping robot

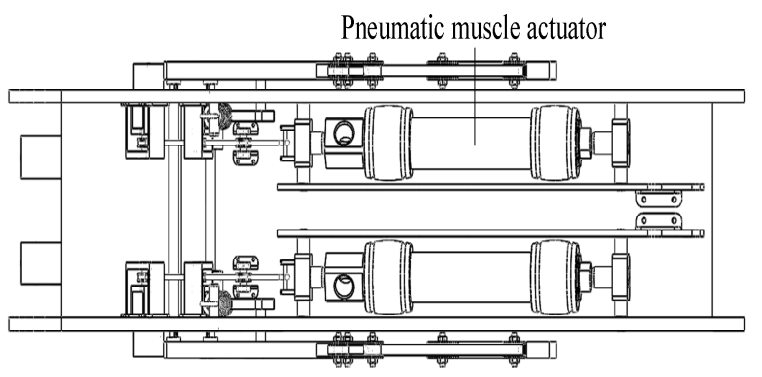

Figure 2 Arrangement of pneumatic muscle actuators for the hind leg of the robot

The two ends of the pneumatic artificial muscle are connected with the connector 1 and 2 through threads. The sheet metal is connected to the bottom of the locust robot by the fixed part and fixed to the appropriate position. The connector is axially positioned to the locust body and the fixed sheet metal by its shaft shoulder, and the body and the sheet metal have track grooves, and the connecting component are placed in the slot. When the pneumatic artificial muscle is contracted, the connecting component moves along the track and pulls the wire rope, which drive the motion of the power leg.

\section{Simulation and discussion of robot dynamics}

Simulation. Firstly, a simplified model of the locust-inspired robot is constructed in Solidworks software and imported into the ADAMS software (shown in Figure 3). Considering the locust-like robot described in this paper is a two-legged type, and the same constraints are added to the other leg in turn as above, so the prototype makes up a whole. Then it is needed to define the parameters between the robot and the ground. Take component 2 as the ground and it could be added fixed constraints between it and the grid surface to ensure the component 2 be not moved. It is needed to add entity collision between the ground and the component of the foot and the front part, and to adjust friction coefficient to simulate the contact between the component and the ground in the actual movement. Entity collision constraints should be added to each member to prevent rod movement from exceeding its limited position during jumping. Component 7 is fixed to the body by adding a fixed pair between the component 7 and the body. At the same time, an entity collision constraint is required to be added to the member 2 and the component 7 to prevent rod 2 from exceeding its limited position when it resets. The jumping process of the bionic locusts is simulated by ADAMS environment. The simulation time is set to $0.8 \mathrm{~s}$, and the number of simulation steps is set to 500 steps, which means simulating the motion process of the jumping robot in $0.8 s$, and decompose jumping process into 500 steps. The simulated jumping process is shown in Figure 4.

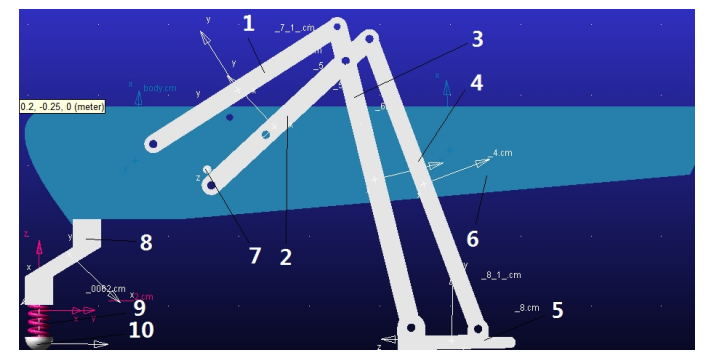

Figure 3 The whole model of ADAMS simulation of bionic locusts

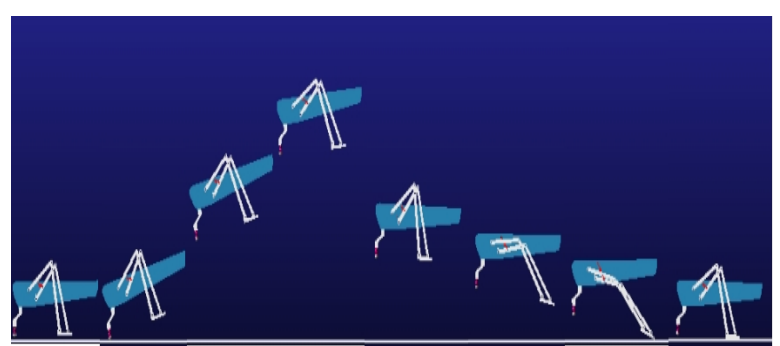

Figure 4 Decomposition diagram of jumping process 
Discussion. The bionic locust's kick action will cause static friction between the legs and the ground, and the counter force of static friction forces the robot to jump forward. The distance of its leap is shown in Figure 5. Its peak represents the longest distance of the jump which is about $1.25 \mathrm{~m}$.

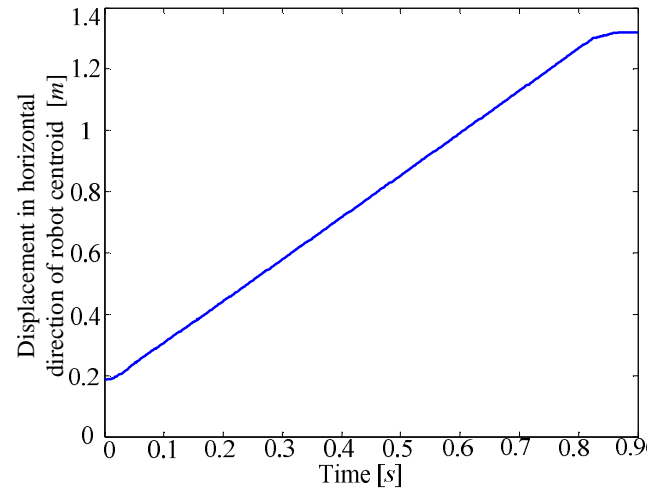

Figure 5 Centroid displacement in horizontal direction

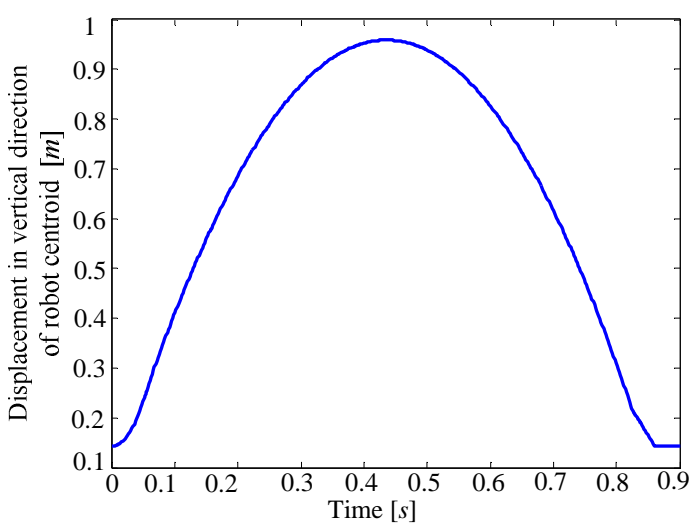

Figure 6 Centroid displacement in vertical direction

The height of robot jumping is an important reference index for evaluation of jumping robot. The height changes in vertical direction during simulation are shown in Figure 6. Under the action of gravity, the curve is basically a segment of parabola, and its initial value is the initial distance between the selected points.

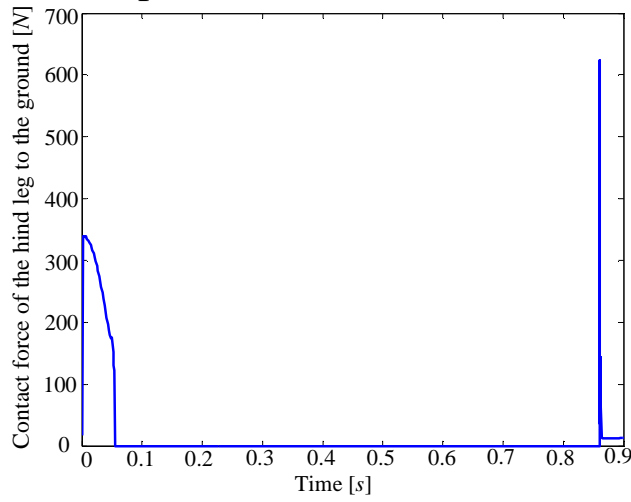

Figure 7 Contact force of the hind leg to ground

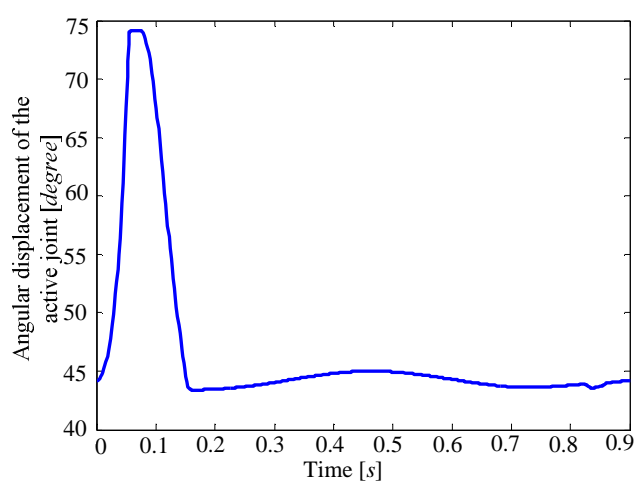

Figure 8 Angular displacement of the active joint

The contact between the hind legs of locust and the ground is particularly important in the process of jumping, which will affect the vertical height and horizontal distance of the final jump. During the jump process, the contact force between the hind legs and the ground will be measured through the measurement function in ADAMS. Its amplitude is shown in Figure 7. The angle change curve of the dynamic leg is shown in Figure 8. The maximum of angle change is about 50 degrees, then it gradually tends to be stable under the function of spring.

\section{Conclusion}

This paper present a novel bionic hopping robot driven by pneumatic muscle actuators. Jumping mechanism of locusts is analyzed and mechanical structure of the robot is designed. Hopping simulation in ADAMS software is performed, and results demonstrate the feasibility of the robot scheme and provide reference for further research of the robot.

\section{Acknowledgments}

This work is supported by Natural Science Foundation of Jiangsu Province (BK20170301); Science and technology planning projects of Changzhou (CJ20179042); The Fundamental Research Funds for the Central Universities (2016B11814). 


\section{References}

[1] Kovač M, Schlegel M, Zufferey J C, et al. Steerable miniature jumping robot[J]. Autonomous Robots, 2010, 28(3):295-306.

[2] Hosoda K, Sakaguchi Y, Takayama H, et al. Pneumatic-driven jumping robot with anthropomorphic muscular skeleton structure[J]. Autonomous Robots, 2010, 28(3):307-316.

[3] Wang T, Guo W, Li M, et al. CPG Control for Biped Hopping Robot in Unpredictable Environment[J]. Journal of Bionic Engineering, 2012, 9(1):29-38.

[4] Zhao J, Xu J, Gao B, et al. MSU Jumper: A Single-Motor-Actuated Miniature Steerable Jumping Robot[J]. IEEE Transactions on Robotics, 2013, 29(3):602-614.

[5] Reis M, Iida F. An Energy-Efficient Hopping Robot Based on Free Vibration of a Curved Beam[J]. IEEE/ASME Transactions on Mechatronics, 2014, 19(1):300-311.

[6] Koh J S, Jung S P, Noh M, et al. Flea inspired catapult mechanism with active energy storage and release for small scale jumping robot[C]// IEEE International Conference on Robotics and Automation. IEEE, 2013:26-31.

[7] Mathis F B, Mukherjee R. Apex height control of a two-mass hopping robot[C]// IEEE International Conference on Robotics and Automation. IEEE, 2013:4785-4790.

[8] Carlési N, Chemori A. Nonlinear model predictive running control of Kangaroo robot: A one-leg planar underactuated hopping robot[C]// Ieee/rsj International Conference on Intelligent Robots and Systems. IEEE, 2010:3634-3639.

[9] Okada M, Takeda Y. Optimal design of nonlinear profile of gear ratio using non-circular gear for jumping robot[C]// IEEE International Conference on Robotics and Automation. IEEE, 2012:1958-1963.

[10] Xiaoxiang Yu, Fumiya Iida. Minimalistic Models of an Energy-Efficient Vertical-Hopping Robot[J]. IEEE Transactions on Industrial Electronics, 2014, 61(2):1053-1062.

[11] Bergbreiter S, Pister K S J. Design of an Autonomous Jumping Microrobot[C]// IEEE International Conference on Robotics and Automation. IEEE, 2014:447-453.

[12] Zhao J, Zhao T, Xi N, et al. MSU Tailbot: Controlling Aerial Maneuver of a Miniature-Tailed Jumping Robot[J]. IEEE/ASME Transactions on Mechatronics, 2015, 20(6):2903-2914.

[13] Terry P, Byl K. A higher order partial feedback linearization based method for controlling an underactuated hopping robot with a compliant leg[C]// Decision and Control. IEEE, 2014:2971-2978.

[14] Zaitsev V, Gvirsman O, Ben H U, et al. A locust-inspired miniature jumping robot.[J]. Bioinspiration \& Biomimetics, 2015, 10(6):066012.

[15] Zhang Z, Zhao J, Chen H, et al. A Survey of Bioinspired Jumping Robot: Takeoff, Air Posture Adjustment, and Landing Buffer[J]. Applied Bionics \& Biomechanics, 2017, 2017:1-22.

[16] Terry P, Piovan G, Byl K. Towards precise control of hoppers: Using high order partial feedback linearization to control the hopping robot FRANK[C]// Decision and Control. IEEE, 2016:6669-6675. 